\title{
Confronting Variation in the Social and Behavioral Sciences
}

\begin{abstract}
I pose problems for the views that human nature should be the object of study in the social and behavioral sciences and that a concept of human nature is needed to guide research in these sciences. I proceed by outlining three research programs in the social sciences, each of which confront aspects of human variation. Next I present Elizabeth Cashdan and Grant Ramsey's related characterizations of human nature. I go on to argue that the research methodologies they each draw on are more productive resources for social scientists than their competing characterizations of human nature.

\section{Acknowledgements}

I would like to thank the participants at our PSA 2014 Symposium session for helpful comments on the presentation this paper was based on, especially Matt Haber, Maria Kronfeldner, Tim Lewens, Edouard Machery and Grant Ramsey. My colleague Jim Tabery and three anonymous referees also made very helpful comments on the manuscript for which I am very grateful.
\end{abstract}

Author Contact: Stephen M. Downes, Department of Philosophy, University of Utah.s.downes@utah.edu 
Copyright Philosophy of Science 2016

Preprint (not copyedited or formatted)

Please use DOI when citing or quoting

Confronting Variation in the Social and Behavioral Sciences

1. Introduction. Philosophers and social scientists defend various accounts of human nature that are labeled non-essentialist. Such accounts are claimed both to characterize the object of inquiry for the social and behavioral sciences and to constrain and guide research (Cashdan, 2013; Griffiths, 2011; Machery, 2008; Ramsey, 2013; Samuels, 2012). The conceptual debate over whether there are defensible non-essentialist accounts of human nature is ongoing (See e.g. Dupre, 1998; Kronfeldner, Roughley, \& Toepfer, 2014; Lewens, 2012, 2015; Machery, 2008, 2012, Forthcoming; Samuels, 2012). Here I present some problems for the view that human nature should be the object of study in the social and behavioral sciences as well as the idea that a concept of human nature is needed to guide or constrain the social and behavioral sciences. I conclude that pursuing a concept of human nature is not a productive approach to confronting human variation. Further, I conclude that some proposed characterizations of human nature are better understood as competing evolutionary approaches to confronting human variation. I begin by outlining three research programs in the social sciences, each of which confront aspects of human variation via what I call a variationist approach. This approach does not rely on an account of human nature.

2. Variationists in the social sciences. Variationists in the social and behavioral sciences accept that variation is pervasive and confront it head on'. They document variation and seek to explain its sources. Variationists come from many fields. Variationist work is more like work in population genetics or evolutionary ecology $y^{2}$ than it is like much work in anthropology or cognitive psychology. In contrast to the variationist approach, much work in cognitive psychology is carried out under the assumption that there are shared, basic psychological capacities that underlie our behavior and characterizing these basic capacities serves an explanatory function. Also, much work in anthropology is carried out under the assumption that there are human universals and the main aim of anthropology is to delineate these universals. Here I outline the work of three variationists in the social sciences.

2.1 Stanovich on reasoning. Much work in the psychology of reasoning is taken to support the conclusion that we are irrational or at least deficient in our reasoning capacities (See e.g. Cohen, 1981; Stich, 1990). Well-known experiments, often used illustratively in philosophy classes, are taken to reveal that we cannot reason deductively very well or that we are susceptible to fallacies such as ignoring base rates. There are a number of hypotheses about

' James Tabery (2014) labels some social science researchers "variationpartitioners" in contrast with "mechanism-elucidators." Variation-partitioners consider the issue of how much variation can be attributed to various candidate causes of variation. The group identified here as "variationist" includes both Tabery's variation-partitioners and mechanism-elucidators along with others in the social sciences who confront and seek to understand human variation. ${ }^{2}$ In this regard, the variationist approach is closely aligned with populationist thinking, which Sober distinguishes from typological thinking (Sober, 1980). 
Copyright Philosophy of Science 2016

Preprint (not copyedited or formatted)

Please use DOI when citing or quoting

what mechanisms underlie our reasoning that would produce such results in experimental situations. One view is that we have an innate deductive reasoning mechanism but, like our innate language capacity, the mechanism gives us a competence but our performance falls down in empirical situations (See e.g. Macnamara, 1986). Stanovich (1999) takes a different approach to the results of the experimentation on reasoning than most of his colleagues in cognitive psychology. He "attempts to foreground a type of empirical data that has been underutilized [...] in the debate about human rationality" data about individual differences $(1999,2)$. He says that parties to the rationality discussion in psychology overemphasize modal responses and ignore patterns of difference in responses, including the occurrence of the correct response in all experiments. Stanovich (1999) presents the study of human reasoning as the study of variation and individual differences in reasoning.

Various forms of the Wason Selection (Wason, 1966; Wason \& JohnsonLaird, 1972) task are used in experiments on deductive reasoning (See figure 1. below). In more abstract versions of the task, such as the card removal task, experimenters find very low percentages of subjects provide the "correct" answer. The "correct" answer in the deductive reasoning literature is the answer that would be obtained by using only valid deductive inference. Stanovich (1999) averages over findings from a number of abstract Wason style selection tasks and finds that around $10 \%$ of subjects provide the correct answer. On selection tasks with more contextual cues, subjects perform much better but a significant percentage of subjects still do not provide the "correct" answer. Stanovich performs various comparative studies in an attempt to find patterns in the variation. For example, he compares subjects' performance on SAT tests with their performance on various reasoning tests.

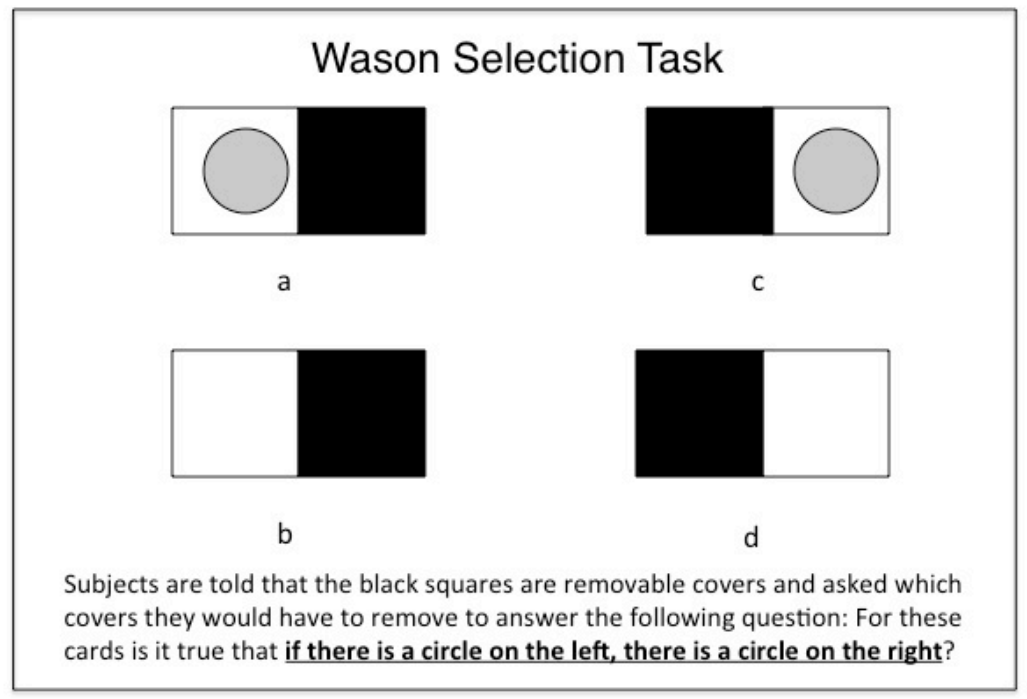

Figure 1. 
Copyright Philosophy of Science 2016

Preprint (not copyedited or formatted)

Please use DOI when citing or quoting

2.2 Henrich and WEIRD people. Many in the cognitive sciences proceed on the assumption that there are basic psychological capacities, whose existence is demonstrated by experimental work. Joe Henrich and his colleagues (2010) argue that there is far less support for basic psychological capacities if experiments are performed on subjects from countries other than western, educated, industrialized, rich and democratic (WEIRD) countries. The bulk of psychological experimentation is performed on subjects from WEIRD countries and Henrich et al. question why these subjects should be presented as "representative of the species" as opposed subjects from any other population. In a recent study Henrich and his collaborators (2010) collect results from experiments performed on subjects from non-WEIRD countries and compare them to the results from the same experiments on subjects from WEIRD countries. What they reveal is a great deal of variation in subject responses. They conclude "members of WEIRD societies, including young children, are among the least representative populations one could find for generalizing about humans" $(2010,61)$. They also warn "we need to be less cavalier in addressing questions of human nature on the basis of data drawn from this particularly thin, and rather unusual, slice of humanity" $(2010,61)$.

Henrich et al. present one study that indicates cross cultural variation in our responsiveness to the Müller-Lyre illusion (2010, 64-65). The Müller-Lyre illusion is taken to be extremely robust and thought to reveal underlying constancies in our visual processing. These assumptions contribute to the illusions' important role in Fodor's (1983) argument for the modularity of the visual system. Henrich et al. present the results from Segal et al.'s (1966) cross cultural study on visual perception. Segal et al. tested responses to the MüllerLyre illusion along with several other well-known perceptual illusions, such as the Sander Parallelogram, on subjects from a wide range of cultures (Henrich, 2008). They found that the strongest effect of the Müller-Lyre illusion is on what Henrich et al. now call WEIRD people (See also Henrich, 2008)." Henrich et al. present the results of many other studies that show differences between WEIRD and non-WEIRD subjects' responses to psychological tests. For example, there is evidence of variation in spatial cognition across cultures $(2010,68)$ and variation in the way in which people from different cultures play Ultimatum Games (2010, 65).

2.3 Cashdan and variation in women's waist-hip ratios. A well known evolutionary psychology hypothesis about human mate selection is that men are

${ }^{3}$ Rebecca Dresser (1992) poses and discusses the related question: How did white males become the prototype research subject in the medical sciences?

" There were a few follow-up studies (Davis \& Carlson, 1970; Jahoda, 1966) conducted at around the same time as the Segal et al. study that produced partial replication of the Segal et al. results and challenged Segal et al.'s explanatory hypotheses for the relevant variation. These studies used subjects from one nonWEIRD population and subjects from a WEIRD population but did not repeat the Segal et al. study in the same range of populations (These studies were brought to my attention by Joe Henrich and Edouard Machery, personal communication). 
Copyright Philosophy of Science 2016

Preprint (not copyedited or formatted)

Please use DOI when citing or quoting

attracted to women with a waist-hip ratio (WHR) close to .7 (Singh, 1993; Singh

\& Luis, 1995). Singh et al. proposed that .7 is the optimal WHR as it is the

WHR most prevalent in young women of child-bearing age. Their claim is not just that men are most attracted to women with optimal WHR's but that they have an evolved module for detecting such optimal WHR's and this module is part of the human male mate-selection suite of evolved modules. Evolutionary anthropologist Elizabeth Cashdan (2008) focuses on different aspects of WHR's. She documents a huge amount of variation in WHR. This variation occurs across different dimensions, there is variation among young women, variation among older women as well as variation between different populations. There is also variation in women's lifetimes; in most cases young to old implies low to high WHR. Second, Cashdan (2008) finds an important variable, or set of variables, that might contribute to explaining some of this variation in WHR's: the ratios between women's hormone levels, for example, the ratio of androgen levels to other hormone levels. Finally, Cashdan documents variation in what WHR's men find attractive (Also shown in Yu \& Shepard, 1998). Cashdan (2008) discusses the very interesting result of preferred WHR's increasing during hard times or times of low resource in both Western and more traditional societies. This indicates that men are tracking something other than just low WHR and implies that they do not have an inflexible module for picking out low WHR. In other words, there is variation in what men from different cultures find attractive with respect to WHR and there is variation within subjects in what they find attractive with respect to WHR depending on available resources. Cashdan concludes that likely the least interesting claim in the WHR literature is that men are universally attracted to women with lower WHR's.

What all these variationists have in common is that they attempt to discover patterns in the variation of human traits and propose hypotheses about what could explain these patterns of variation. In this way their work is closer to work in population genetics than to human nature based research in the social and behavioral sciences.

3. Variation sensitive accounts of human nature. I now turn to two accounts of human nature that are developed to confront human variation. They are: Elizabeth Cashdan's norm of reaction account and Grant Ramsey's life-history trait cluster account. First, I will briefly outline Edouard Machery (2008, 2012, Forthcoming) and Richard Samuel's (2012) accounts of human nature as it is useful to contrast them with accounts that emphasize variation.

\subsection{Machery and Samuels' non-essentialist accounts of human nature. Machery} (2008) distinguishes two independent notions of human nature: the essentialist notion, which he rejects along with Hull and others, and the nomological notion, his new proposal. The nomological notion says that "human nature is the set of properties that humans tend to possess as a result of the evolution of their species" (Machery, 2008, 323). On this account bipedalism is part of human nature but supporting Liverpool Football Club is not. Machery refers to this part of his account as the evolutionary proposal but adds that the nomological notion has nothing to do with defining species membership. His nomological account of human nature is not essentialist and is not intended as a proposal for delineating our species. He also maintains that being common among humans is 
Copyright Philosophy of Science 2016

Preprint (not copyedited or formatted)

Please use DOI when citing or quoting

a necessary condition for being part of human nature. He refers to this as the universality proposal of the nomological account. His idea is that traits that arise purely as a result of local cultural circumstances are very unlikely to be common among humans. So his account contains two central proposals the evolutionary proposal and the universality proposal.

Samuels offers a related view to Machery's, which he dubs the "causal essentialist" account of human nature. He says that "human nature is a suite of mechanisms that underlie the manifestation of species-typical cognitive and behavioural regularities" $(2012,2)$. For Samuels human nature picks out a "set of phenomena that will form a focus of empirical enquiry for some region of science" $(2012,4)$. Samuels claims that his characterization of human nature is "a conception on which human nature can play its customary causal-explanatory function" $(2012,18)$ and hence should be adopted over Machery's nomological account.

3.2. Cashdan's norm of reaction account of human nature. Cashdan, like many evolutionary thinkers, emphasizes variation (See her 2008 discussed above). Cashdan's approach to anthropology is grounded in behavioral ecology. Behavioral ecologists strive to understand and explain behavior without resorting to appeals to underlying psychological mechanisms or underlying genetic systems. Behavioral ecologists focus on relations between organisms' behaviors and aspects of their environments. Anthropologists relying on this approach have presented and defended interesting hypotheses about human parenting, food acquisition and distribution and variation in age specific traits. In contrast, competing hypotheses from evolutionary psychology about the same phenomena are be couched in terms of shared, evolved, internal psychological mechanisms that are causally responsible for the relevant behavior. This approach is rejected by behavioral ecologists who emphasize variation over commonality.

Cashdan rejects the assumption, held by many in her field of anthropology, that "human nature is found solely in its universals - in the traits found in every society" $(2013,71)$. Those who hold this assumption (e.g. Brown, 1991) go onto say that traits found in some cultures but not others are "culturally constructed and without an evolutionary foundation" $(2013,71)^{\text {s. In contrast }}$ Cashdan grounds her approach on the assumption that we evolved to be flexible. She goes on to propose that we ask how natural selection shaped that flexibility. She says that "we cannot understand our universal human nature without understanding the variability in its expression" $(2013,71)$. Cashdan does aim to reveal our nature but argues that our nature is neither a set of underlying causal mechanisms (cf. Samuels, 2012) nor the collection of traits that we have as a result of evolution (cf. Machery, 2008). Rather, our nature is to be found in patterns of variation. Cashdan proposes to reveal these patterns in variation by invoking norms of reaction, which are "the pattern of expression of a genotype across a range of environments" $(2013,71)$. Norms of reaction are standardly presented by plotting the relation between a trait value and an environmental factor for specific genotypes (See Figure 2. below). According to Cashdan, all the

${ }^{5}$ This distinction is clearly maintained in Machery's (2008) account of human nature (See also Machery, Forthcoming). 
Copyright Philosophy of Science 2016

Preprint (not copyedited or formatted)

Please use DOI when citing or quoting

reaction norms for all our genes in all environments together constitute our nature.

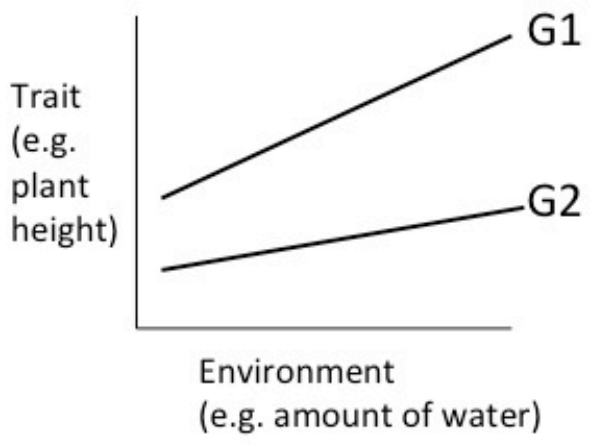

Figure 2.

3.3. Ramsey's life history trait cluster account of human nature. Ramsey (2013) sets up his account of human nature by responding to Machery's nomological account of human nature. He asks "why should we presume that it is the sameness across individuals that is of interest to scientists, and not their variation?" $(2013,986)$. He goes on to conclude his first argument against Machery by saying: "it is a mistake to hold that only traits universal (or nearly universal) in the human species are of scientific interest and should be included within human nature" $(2013,986)$ Here he echoes Cashdan's criticism of her colleagues' over-emphasis on universals in anthropology. For Ramsey, like Cashdan, our focus in the social sciences should be accounting for variation. He proposes to do this guided by a non-essentialist account of human nature that he believes improves upon Machery's nomological account.

For Ramsey "individual nature is defined as the pattern of trait clusters within the individual's set of possible life histories" and "human nature is defined as the pattern of trait clusters within the totality of extant human possible life histories" $(2013,987)$. He calls this the life-history trait cluster (LTC) account of human nature. Different possible life histories for organisms result from the range of possible developmental responses organisms make to differing environmental circumstances. He also proposes that "characterizations of 
Copyright Philosophy of Science 2016

Preprint (not copyedited or formatted)

Please use DOI when citing or quoting

features of human nature are merely descriptions of patterns within the

collective set of human life histories" $(2013,988)$. According to Ramsey, the LTC framework shows that "there are patterns within and across human heterogeneity" $(2013,992)$.

There are others who defend accounts of human nature that encompass varaiation. For example, Clark Barrett (2015) defends a related notion of human nature to Cashdan and Ramsey's, proposing that human nature is the sum total of variation in our lineage. Also, Paul Griffiths (2011) defends a notion of human nature derived from Developmental Systems Theory and aimed at accounting for the whole range of human diversity. The details of Cashdan and Ramsey's accounts given here will suffice to support the arguments in the next section that apply to all defenders of biological accounts of human nature.

4. Accounts of human nature and confronting variation. Characterizations of human nature are proposed to set both the object of inquiry in the social sciences and to guide that inquiry. Alternately, characterizations of human nature can be seen as serving various functions, the most relevant here being a descriptive or an explanatory function'. Cashdan, Ramsey's and related characterizations of human nature all have the same problem: each account amounts to the assertion that an enormous collection of traits constitute human nature. This type of account is anticipated by various critics of human nature who argue that such accounts are not theoretically interesting (See e.g. Buller, 2005; Futuyma, 1998; Hull, 1986). I apply these criticisms to Cashdan and Ramsey below and also argue that these criticisms can be recast to make the point that the relevant accounts of human nature fail to perform an explanatory function.

Cashdan and Ramsey propose that all of the traits that can arise as a result of our life long interaction with our environments constitute human nature. Their approaches are structurally very close to David Hull's disjunctive notion of universality, which he invokes as an account of human nature that might be proposed to confront variation. Hull uses the example of blood type: "blood type can be made universal among human beings only by defining it in terms of having some blood type of other - a disjunctive character" (Hull, 1986, 5). Hull calls this move "universality on the cheap." The idea here is that simply expanding the list of traits in our nature disjunctively does not result in an explanatorily useful notion of human nature. While it is certainly true that we can discover a wide variety of human traits, and that a norm of reaction approach or Life History Theory help us understand just how wide that variety of traits may be, drawing a line around all of these possible traits does not result in a theoretically useful characterization of human nature. An explanatorily useful notion of human nature should be expected to shed light on the variation in human reasoning, waist hip ratios, or human cognitive function outlined above in Section 2. What Cashdan and Ramsey's accounts can offer is the observation that all this variation is part of human nature and this alone, does not provide any explanatory purchase on all of this variation.

Most of Cashdan and Ramsey's critical points against rival human nature accounts such as Machery's and Samuels' are on target. For example, claims that

' See Samuels (2012) and Machery (Forthcoming) for more on the various functions characterizations of human nature should serve. 
Copyright Philosophy of Science 2016

Preprint (not copyedited or formatted)

Please use DOI when citing or quoting

we should not conceptualize human nature in terms of universality, we should emphasize variation over similarity and we should not limit our accounts by including only internal properties of organisms all have merit. Their criticisms of alternate accounts of human nature are undermined by their presentation of their own approaches as alternate characterizations of human nature. As we have seen, their accounts have drawbacks. Further, their accounts of human nature do not provide guides for research in social and behavioral sciences. Rather, their positive accounts are all best understood as alternate, important and productive approaches to studying human variation derived from different areas of evolutionary thought. They present and defend alternate clusters of evolutionary methods and explanatory assumptions that can be productively applied by social and behavioral scientists confronting human variation. Life History Theory or a Norm of Reaction approach could shed important light on variation in human reasoning capacities, waist-hip ratios or depth perception. In contrast, accounts of human nature in terms of variation are not likely to increase our understanding of these phenomena. The valuable explanatory potential in Cashdan and Ramsey's accounts comes not from their accounts of human nature but from the alternate evolutionary resources they draw upon in constructing their accounts.

There is a very tight relationship between each characterization of human nature outlined above and distinct clusters of methods and explanatory assumptions. As we have seen, Cashdan and Ramsey both characterize human nature in terms of different approaches in evolutionary biology. Machery and Samuels also explicitly align their characterizations of human nature with alternate explanatory approaches in the social sciences. Machery's nomological notion is designed to be consistent with the research methodology of evolutionary psychology, and to some extent sociobiology $(2008,328)$, and Samuel's notion is designed to be consistent with the research methodology of cognitive psychology and cognitive neuroscience $(2012,27)$. Perhaps Samuels does not have variation in his sights because cognitive psychologists' primary focus is not on variation. Stanovich and Henrich's work, discussed above, can be viewed as an attempt to change the focus of their cognitive science colleagues towards variation but, this work not withstanding, Samuels is right that the main focus in the cognitive sciences is on revealing shared underlying cognitive mechanisms. In contrast, Machery, like Cashdan and Ramsey, draws on an avowed evolutionary approach and one central aim of evolutionary biology is the delineation of variation and the explanation its causes (cf. Hull, 1986, 5). The puzzle here then is why adopting an evolutionary approach points Machery towards commonality rather than variation.

Machery draws on an approach to evolutionary psychology articulated and defended by Leda Cosmides and John Tooby (See e.g. Tooby \& Cosmides, 1990, 2005) among others. Cosmides and Tooby draw an illuminating contrast between evolutionary psychology and behavioral genetics $(2005,39)$. They argue that while behavioral geneticists focus on traits that vary, evolutionary psychologists focus on traits that are now universal and do not vary. These traits are the product of evolutionary processes that occurred during the Pleistocene 
Copyright Philosophy of Science 2016

Preprint (not copyedited or formatted)

Please use DOI when citing or quoting

era. Machery does not have to adopt this approach to the letter to still claim that evolutionary biologists confront and account for both traits that vary and traits that vary very little (cf. Lewens, 2015). This is true, evolutionary dynamics account for both the fixing of traits and the sustenance of variation. The problem here is that contrary to Cosmides and Tooby's assumption, human traits that vary sufficiently minimally and are widely enough distributed to be included in Machery's nomological human nature cluster are unlikely to lead to interesting and useful explanations of human traits of interest to social scientists. It is hard to establish that cognitive mechanisms are universal adaptations (cf. Buller, 2005; Henrich et al., 2010) but we can point to highly invariant human traits such as bipedalism and the size of calcium ion channels are both highly invariant human traits but neither provide an obvious basis for productive explanations in the social and behavioral sciences.

All the characterizations of human nature discussed above closely track different methodological approaches. Cashdan, Machery and Ramsey all propose a characterization of human nature that draws heavily upon their favored evolutionary approach. What these approaches all have in common is to offer alternate evolutionary approaches to social scientists but only the approaches offered by Cashdan and Ramsey will help in the quest to account for and explain human variation.

5. Conclusion. Adopting a variationist approach is the more productive strategy in the social and behavioral sciences than the search for human universals or traits in common that constitute our nature. Cashdan and Ramsey agree with this outlook as they all agree that the explanatory target of each of their approaches should be human variation. Cashdan and Ramsey propose fruitful ways of explaining human behavior but I argued that their most fruitful contributions are not their alternate characterizations of human nature. Rather, they each provide alternate, evolutionarily influenced, frameworks for understanding and explaining human variation, both of which are valuable resources for social scientists confronting human variation. In contrast, Machery backs an evolutionary approach that does not contribute to understanding and explaining human variation.

There appear to be almost as many notions of human nature as there are clusters of methods and explanatory assumptions in the social and behavioral sciences and I take this to undermine the idea that there should be one notion of human nature rather than support it. Productive social and behavioral science is a broad based interdisciplinary project. Participants in this project would have plenty of important and productive work left to do if they abandoned the quest for an account of human nature and focused on human variation and the attempt to account for why it arises and is sustained throughout human populations.

- Barrett (2015) promotes an approach to evolutionary psychology that does focus on variation and criticizes his colleagues for uncritically assuming that universal traits should be the focus of evolutionary psychology. 
Copyright Philosophy of Science 2016

Preprint (not copyedited or formatted)

Please use DOI when citing or quoting

\section{REFERENCES}

Barrett, H. C. (2015). The Shape of Thought: How mental adaptations evolve. Oxford: Oxford University Press.

Brown, D. E. (1991). Human Universals. New York: McGraw-Hill.

Buller, D. (2005). Adapting Minds: Evolutionary Psychology and the Persistent Quest for Human Nature. Cambridge, MA: MIT Press.

Cashdan, E. (2008). Waist-to-Hip Ratio across Cultures: Trade-offs between Androgen- and Estrogen-Dependent Traits. Current Anthropology, 49(6), 10991107.

Cashdan, E. (2013). What is a human universal? Human behavioral ecology and human nature. In S. M. Downes \& E. Machery (Eds.), Arguing About Human Nature (pp. 71-80). New York: Routledge.

Cohen, L. J. (1981). Can human irrationality be experimentally demonstrated?

Behavioral and Brain Sciences, 4, 317-370.

Davis, C. M., \& Carlson, J. A. (1970). A cross-cultural study of the strength of the Muller-Lyer illusion as a function of attentional factors. Journal of Personality and Social Psychology, 16(3), 403-410.

Dresser, R. (1992). Wanted Single, White Male for Medical Research. The Hastings Center Report, 22(1), 24-29.

Dupre, J. (1998). Normal People. Social Research, 65(2), 221-248.

Fodor, J. A. (1983). The Modularity of Mind. Cambridge, MA: MIT Press.

Futuyma, D. J. (1998). Evolutionary Biology (Third ed.). Sunderland, MA: Sinauer. Griffiths, P. E. (2011). Our Plastic Nature. In S. B. Gissis \& E. Jablonka (Eds.),

Transformations of Lamarckism: From subtle fluids to Molecular Biology (pp. 319-330). Cambridge, MA: MIT Press.

Henrich, J. (2008). A cultural species. In M. Brown (Ed.), Explaining Culture Scientifically (pp. 184-210). Seattle: University of Washington Press.

Henrich, J., Heine, S., \& Norenzayan, A. (2010). The weirdest people in the world. Behavioral and Brain Sciences, 33, 61-83.

Hull, D. L. (1986). On Human Nature. PSA:Proceedings of the Biennial Meeting of the Philosophy of Science Association, 2, 3-13.

Jahoda, G. (1966). Geometric illusions and environment: A study in Ghana. British Journal of Psychology, 57(1-2), 193-199.

Kronfeldner, M., Roughley, N., \& Toepfer, G. (2014). Recent work on human nature: Beyond traditional essences. Pihlosophy Compass, 9, 642-652.

Lewens, T. (2012). Human Nature: The Very Idea. Philosophy \& Technology, 25, 459-474.

Lewens, T. (2015). Cultural Evolution. Oxford: Oxford University Press.

Machery, E. (2008). A Plea for Human Nature. Philosophical Psychology, 21, 321329.

Machery, E. (2012). Reconceptualizing Human Nature: Response to Lewens. Philosophy \& Technology, 25, 475-478.

Machery, E. (Forthcoming). Human Nature. In D. Livingstone_Smith (Ed.), How Biology Shapes Philosophy. Cambridge: Cambridge University Press.

Macnamara, J. (1986). A Border Dispute: The place of logic in psychology. Cambdrige, MA: MIT Press. 
Copyright Philosophy of Science 2016

Preprint (not copyedited or formatted)

Please use DOI when citing or quoting

Ramsey, G. (2013). Human nature in a post-essentialist world. Philosophy of Science, 80, 983-993.

Samuels, R. (2012). Science and Human Nature. Royal Institute of Philosophy Supplement, 70, 1-28.

Segal, M. H., Campbell, D. T., \& Herskovits, M. J. (1966). The influence of culture on visual perception. Indeanapolis: Bobbs_Merrill.

Singh, D. (1993). Adaptive Significance of female physical attractiveness: Role of waist-to-hip ratio. Journal of Personality and Social Psychology, 65, 293-307.

Singh, D., \& Luis, S. (1995). Ethnic and gender consensus for the effect of waist to hip ratio on judgments of women's attractiveness. Human Nature, 6, 51-65.

Sober, E. (1980). Evolution, Population Thinking and Essentialism. Philosophy of Science, 47, 350-383.

Stanovich, K. (1999). Who Is Rational? Studies of individual differences in reasoning. Mahwah, NJ: Lawrence Erlbaum Associates.

Stich, S. P. (1990). The Fragmentation of Reason. Cambridge, MA: MIT Press.

Tabery, J. (2014). Beyond Versus: The Struggle to Define the Interaction of Nature and Nurture. Cambridge, MA: MIT Press.

Tooby, J., \& Cosmides, L. (1990). On the universality of human nature and the uniqueness of the individual. Jounral of Personality, 58, 17-67.

Tooby, J., \& Cosmides, L. (2005). Conceptual Foundations of Evolutionary Psychology. In D. Buss (Ed.), The Handbook of Evolutionary Psychology (pp. 5-67). Hoboken, NJ: Wiley.

Wason, P. C. (1966). Reasoning. In B. Foss (Ed.), New Horizons in Psychology (pp. 135-151). Harmonsworth, UK: Penguin.

Wason, P. C., \& Johnson-Laird, P. N. (1972). Psychology of Reasoning. Cambridge, MA: Harvard University Press.

Yu, D. W., \& Shepard, G. H. (1998). Is beauty in the eye of the beholder? Nature, $396,321-322$. 